\title{
Summer and early-fall sea-ice concentration in the Ross Sea: comparison of in situ ASPeCt observations and satellite passive microwave estimates
}

\author{
Margaret A. KNUTH, ${ }^{1}$ Stephen F. ACKLEY ${ }^{2}$ \\ ${ }^{1}$ Department of Civil and Environmental Engineering, Clarkson University, PO Box 5712, Potsdam, NY 13699-5710, USA \\ E-mail: knuthma@gmail.com \\ ${ }^{2}$ Earth and Environmental Sciences, University of Texas at San Antonio, 1 UTSA Circle, San Antonio, TX 78249, USA
}

\begin{abstract}
Sea-ice conditions were observed using the ASPeCt observation protocol on three cruises in the Ross Sea spanning the Antarctic summer season (APIS, December 1999-February 2000; AnSlope 1, March-April 2003; AnSlope 2, February-April 2004). An additional dataset was analyzed from helicopter video surveys taken during the APIS cruise. The helicopter video was analyzed using two techniques: first, as an ASPeCt dataset where it was sampled visually for ice concentration, floe sizes and ice type on a point basis at $11 \mathrm{~km}$ intervals; second, computerized image processing on a subset of nine helicopter flights to obtain ice concentration on a continuous basis ( $1 \mathrm{~s}$ intervals) for the entire flight. This continuous sampling was used to validate the point-sampling methods to characterize the ice cover; the 'ASPeCt sampling' on the helicopter video and the use of the ASPeCt protocol on the ship surveys. The estimates for average ice concentration agreed within $5 \%$ for the continuous digitized data and point sampling at $11 \mathrm{~km}$ intervals in this comparison. The ship and video in situ datasets were then compared with ice concentrations from SSM/I passive microwave satellite data derived using the Bootstrap and NASA-Team algorithms. Less than $50 \%$ of the variance in summer ice concentration observed in situ was explainable by satellite microwave data. The satellite data were also inconsistent in measurement, both underestimating and overestimating the concentration for summer conditions, but improved in the fall period when conditions were colder. This improvement was in the explainable variance of $>\mathbf{7 0} \%$, although in situ concentration was underestimated (albeit consistently) by the satellite imagery in fall.
\end{abstract}

\section{INTRODUCTION}

Since the launch of the Nimbus-5 electrically scanning microwave radiometer (ESMR) in 1972, passive microwave satellite remote sensing has been used to provide scientists with sea-ice coverage data. Particularly in the case of the polar regions, where obtaining in situ data is a difficult process, passive microwave sensors, unhampered by cloud cover and darkness, are particularly well suited to obtain a significantly large spatial and temporal record of events. The data acquisition was taken over by the scanning multichannel microwave radiometer (SMMR) from 1978 to 1987 and then by the Special Sensor Microwave/Imager (SSM/I) from 1987 to the present. This long dataset gives us an opportunity to understand sea-ice variability on seasonal, interannual and longer timescales. Thus, the passive microwave data provide valuable observations on a daily basis of the global sea ice as it pertains to the climatic response of sea-ice concentration and extent (Worby and Comiso, 2004). Also, the heat and momentum fluxes at the boundary of the atmosphere and ocean significantly depend on the ice concentration. Maps of ice coverage are also important for studies of the regional flora and fauna and for ship navigation.

The satellite data, derived by two well-known algorithms, used for comparison in this work are archived at the US National Snow and Ice Data Center (NSIDC). The two algorithms, NASA-Team (D.J. Cavalieri and others) and Bootstrap (J. Comiso) (http://nsidc.org:80/data/nsidc-0002.html), were both developed at NASA's Goddard Space Flight
Center. While both of these algorithms use brightness temperatures, they use different channels and techniques to produce the total sea-ice concentration. Some work has been done to validate the algorithms, and results have shown that they both have difficulty recognizing newly forming, or thin, sea ice and that snow cover can, at times, mask the signature of the underlying ice (Markus and Cavalieri, 1998; Massom and others, 1999). There are problems particularly during the summer months when the emissivity of the ice is highly variable, and thus finding an appropriate tie point is difficult. Steffen and Schweiger (1991) also showed that the NASATeam algorithm had a larger error in the summer, under melting conditions, and Comiso and others (1997) have also noted that there are large differences between the two algorithms due to discrepancy in temperature and emissivity effects and tie-point differences. However, based on their study of data from 1992, the differences between the two algorithms appear to be smallest during the summer.

The field observations acquired by utilizing the Antarctic Sea Ice Processes and Climate (ASPeCt) protocol while aboard the RVIB Nathaniel B. Palmer and by digital video data retrieved from helicopter flights from the ship are used here to provide some quantification of the errors in ice concentration from summer satellite microwave data and some understanding of the processes that may contribute to these errors. The three cruises took place in the Ross Sea. This area consistently retains a large expanse $\left(1-2 \times 10^{6} \mathrm{~km}^{2}\right)$ of the residual sea ice in the summer, when total sea-ice coverage around Antarctica declines to nearly 20\% of its annual maximum (Gloersen and others, 1992). In this work, 


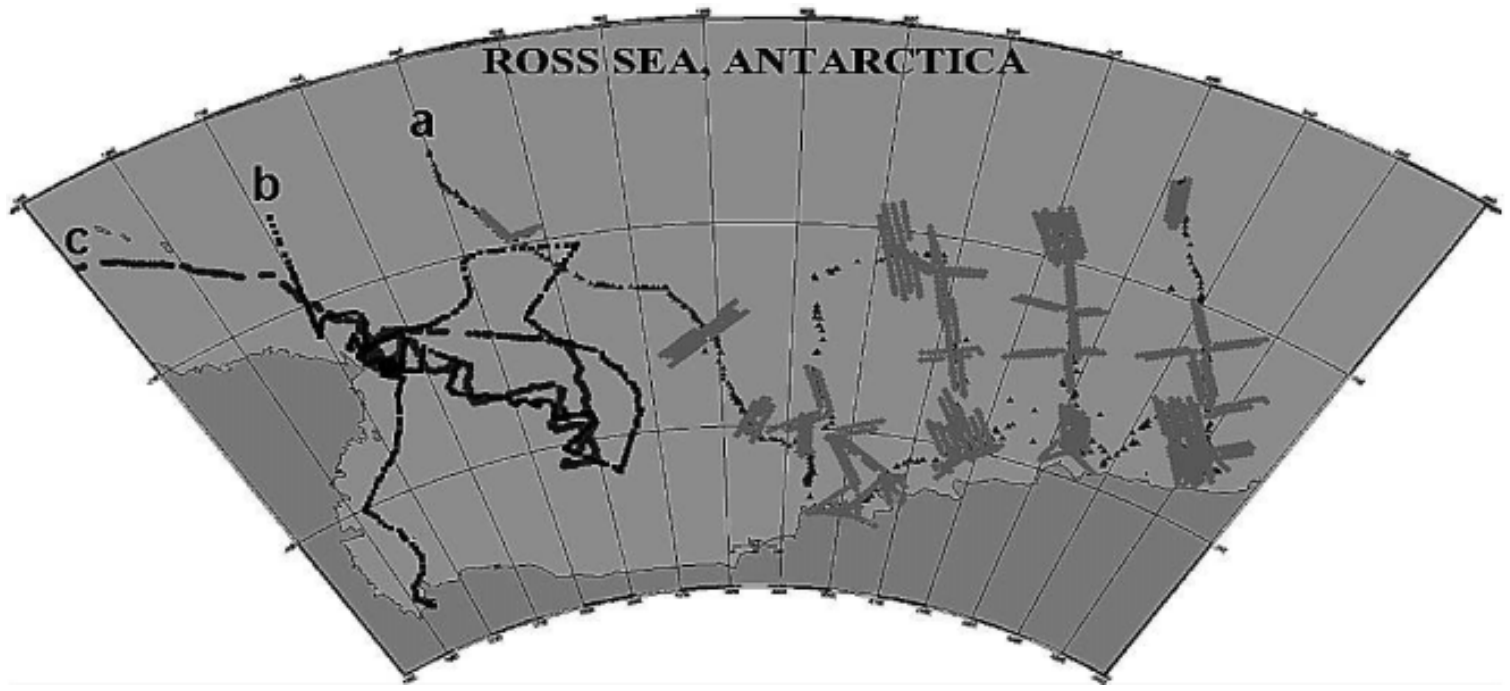

Fig. 1. Cruise tracks for (a) APIS (December 1999-February 2000) (eastern Ross Sea) with helicopter tracks parallel and/or perpendicular to the cruise tracks. Also, in the western Ross Sea, (b) AnSlope 1 (March-April 2003) and (c) AnSlope 2 (February-April 2004) originating from McMurdo Station.

the ice concentrations are compared. A possible relationship between extreme errors (as determined from ice concentration comparison) and variations in other parameters including ice type, snow cover, floe size and surface flooding is also examined.

\section{DATA}

\subsection{Ship data}

The shipboard observations were completed using the protocol specified by the ASPeCt program (Worby and Ackley, 2000). At $11 \mathrm{~km}$ intervals ( hourly), sea ice is observed within a $1 \mathrm{~km}$ radius of the ship. Ice types, total ice concentration and, for each ice type, partial ice concentration, ice thickness, floe size, topography and snow-cover characteristics are estimated. Ice concentration is measured to the nearest $10 \%$ and it is estimated that error in total and partial ice concentrations (due to rounding and observer error) is $10 \%$ (Worby and Comiso, 2004). This protocol was used to characterize the sea ice during three research cruises in the Ross Sea (Fig. 1). The first, from December 1999 to February 2000, was the US component of the international Antarctic Pack Ice Seals (APIS) program, and took place primarily in the eastern region of the Ross Sea. The second dataset is from the first leg of AnSlope (March-April 2003), a cruise looking at cross-slope exchanges at the Antarctic slope front. The third is from the second leg of the AnSlope program (February to April 2004). Both of these cruises were in the western region of the Ross Sea.

\subsection{Helicopter video data}

From 28 December 1999 to 7 February 2000, about 60 helicopter flights were taken from the ship during the APIS cruise. A digital video camera mounted under the helicopter recorded the ice below. Although the main purpose of the helicopter flights was seal reconnaissance, dependable evaluation of the videos for sea ice is possible because of the consistent flight altitude $(\sim 100 \mathrm{~m})$ and speed ( $\sim 85$ knots $\left.\left(\sim 160 \mathrm{~km} \mathrm{~h}^{-1}\right)\right)$ on all flights. Over $18000 \mathrm{~km}$ (track line) was covered and analyzed.
Because this extensive coverage resulted in approximately $20060 \mathrm{~min}$ videos, each video was examined and data were transcribed for $1 \mathrm{~min}(\sim 1.416 \mathrm{~nm})$ at $6 \mathrm{~nm}$ intervals for ice concentration, floe size, ice type and surface ridging area. Because we are observing from helicopter videos instead of the usual platform of a ship, we call this a 'modified' ASPeCt method. It should also be noted that during evaluation we are comparing an average concentration from one entire flight to an average of as many daily averaged satellite pixels that the flight path crosses. This comparison is different from those done in the past. Previous work compared only ice concentration from single images (e.g. from Landsat and Advanced Very High Resolution Radiometer (AVHRR) images), with corresponding pixels from satellite microwave imagery. The present study takes advantage of several larger datasets and compares them to corresponding microwave results.

Helicopter video data were also digitally processed. Nine tapes representing a variety of ice types and concentrations were analyzed. Only nine were evaluated because of the large amount of time it took to complete this process. First, each digital video was uploaded to the computer. There the video was turned into 2500-3000 snapshots that resulted from taking 1 frame s$^{-1}(0.2 \mathrm{~km} \times 0.2 \mathrm{~km})$ from the video. The next step was to make each snapshot binary. Since the video varied from light or dark cloudy conditions to glaringly bright sunny conditions over two highly reflective surfaces (ice and calm water), it was impossible to pick a single brightness threshold to distinguish ice from water and run the snapshots through a common script to make them binary. Therefore, each snapshot was separated by hand into one of four categories: water, full ice cover, partial ice cover, and bright from specular reflection. The last category mainly occurred over the water and not the ice cover. An ice brightness threshold was found for each of these cases and the snapshots were then run through a script in Matrox Inspector to make them binary and to calculate the total ice area of each frame. For the nine tapes analyzed digitally, Figure 2 is a comparison of ice concentration derived from video data by the two different methods. The standard deviation between the two estimates 
is $\pm 3.3 \%$. Using the best fit, from the linear regression line, ASPeCt point sampling generally underestimates the average ice concentration of the digitally derived data (i.e. the continuous or 'real' data) by $2-4 \%$. Both the standard deviation and the predicted concentration are well within the $10 \%$ error previously attributed to the ASPeCt sampling method (Worby and Comiso, 2004). These results indicate that the 'modified' ASPeCt method is an acceptable way to analyze the video data for the entire $18000 \mathrm{~km}$ track line. Henceforth the digitally derived helicopter data will not be used, only those resulting from the 'modified' ASPeCt observations.

\subsection{Satellite data}

The gridded sea-ice concentrations for this work are from the US Defense Meteorological Satellite Program's (DMSP) $\mathrm{SSM} / \mathrm{I}$. The large difference in emissivity between calm water and sea ice is what makes the measurement of sea ice using passive microwave data possible. Daily averaged data, gridded with a resolution of $25 \times 25 \mathrm{~km}$, are acquired from several satellite passes throughout the course of a day. The SSM/I is a seven-channel, four-frequency system that measures surface brightness temperatures. The NASA-Team algorithm uses the vertically polarized 19.4 and $37.0 \mathrm{GHz}$ channels and the horizontally polarized $19.4 \mathrm{GHz}$ channel. It can differentiate between two radiometrically different types of sea ice and open water. The Bootstrap algorithm uses only the vertically polarized 19.4 and $37.0 \mathrm{GHz}$ channels and can only make a distinction between ice and open water. It has been documented that both algorithms have difficulty recognizing areas of new ice and summer melting conditions over sea ice (Steffen and Schweiger, 1991; Markus and Cavalieri, 1998; Massom and others, 1999). The variable emissivity of sea ice during the summer is a particular problem for this study, as we discuss later. This is not the case for the AnSlope cruises that occurred later in the year; nevertheless the AnSlope data represent only about one-third of the total data.

The analysis compares the satellite data to all four of the field datasets combined, i.e. the three ship and one helicopter datasets. Also, in all graphs, 'observed concentration' refers to all of these four surveys combined.

\section{ANALYSIS}

\subsection{All four datasets}

Because of the formats of the satellite, helicopter and ship data, there is a large difference in spatial resolution. Each $1 \mathrm{~min}$ video reading is recorded as an average that covers approximately $0.2 \times 2.6 \mathrm{~km}$, and each shipboard reading is taken from a circle with a $1 \mathrm{~km}$ radius, but they are both compared to a $25 \times 25 \mathrm{~km}$ averaged pixel from the satellite data, i.e. $<1 \%$ of the area of a satellite pixel. Ice concentration is recorded on a scale of $0-100 \%$ coverage. Plotting each value (not shown) results in an extreme scatter of the data, with little evident relationship. To better compare seaice coverage between the three resolutions, we introduced some averaging into the analysis of the field data. Throughout the cruise, the helicopter data were separated into a range of events (one flight per tape) that ranged from 1 to 12 observations per flight event. These events provided an easy way to break up the data for averaging. Shipboard measurements are taken every hour when the ship is in

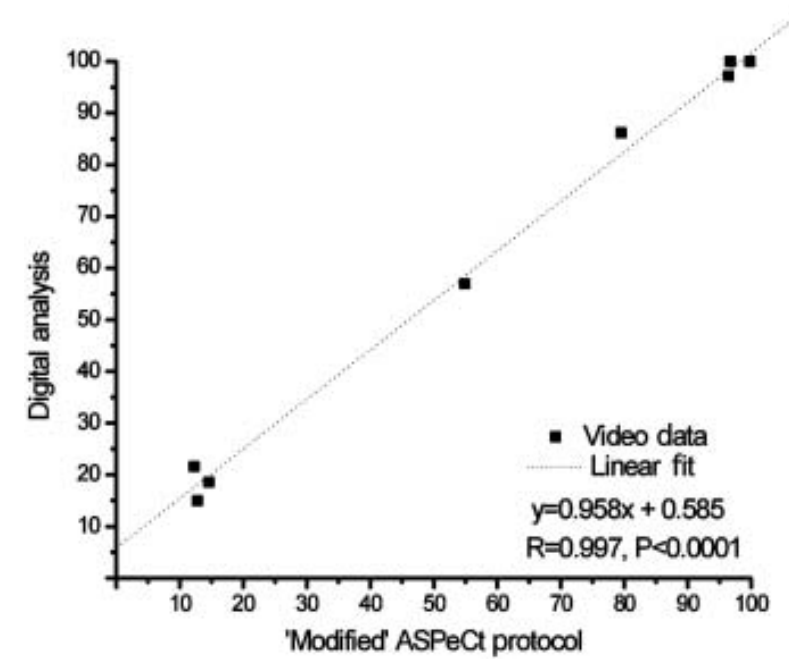

Fig. 2. Comparison of ice concentrations derived from helicopter data. As sampled using the 'modified' ASPeCt protocol ( $x$ axis) vs digitally analyzing the entire tape ( $y$ axis). The linear fit (and equation of the line) is shown. Standard deviation between the two estimates is $\pm 3.3 \%$.

motion, so each day was split up into two 'events', with one occurring from 0 to 11 UTC (Universal Time Coordinated) and the other from 12 to 23 UTC. This also allowed us to have 1-12 observations per event, similar to the helicopter data. So, for example, if a helicopter flight was 1 hour long, it would have approximately twelve observations. If it was $30 \mathrm{~min}$ long, there may only be six observations. Or, if the ship stopped for some work (conductivity-temperaturedepth measurements, mooring recovery, etc.) then there may only be four ship observations in a 12 hour period. (The data discussed here represent those over drifting pack ice. Fastice values from the helicopter video were separated out and are discussed below.)

Since the data were averaged over a variable number of observations, it is important to see if this causes a significant difference in the results. An average concentration was found for each event (flight or half-ship day), and this value was compared with an average concentration derived from the satellite pixels crossed during that particular event. As shown in Figure 3, the data were separated into 1-3, 4-6, 7-9 and 10-12 observations per event. The linear fits for 4-6, 7-9 and 10-12 all have similar $R$ values equal to $\sim 0.7$, and all cross the $y$ axis at a concentration of approximately $15 \%$. The data represented by 1-3 observations per event are much more scattered, with a significantly smaller $R(0.43)$. Looking more closely at other characteristics of these data points, i.e. ice type, floe size and topography, no similarities that might justify the variance difference other than under-sampling were noted. This was also true for comparisons using the Bootstrap algorithm where the average $R$ value for 4-6, 7-9 and 10-12 observations per event was 0.77 , but for $1-3$ observations it was 0.44 . We note also that the more observations per event the more satellite pixels that are also averaged (up to 5 or 6 for the 12 observations per event). Given the issues in comparing data with only 1-3 observations per event (and correspondingly one or two satellite pixels), these values were removed from the dataset for the ensuing analysis, as well as the fast-ice observations from the helicopter video (video ice concentrations of 100\%). The 

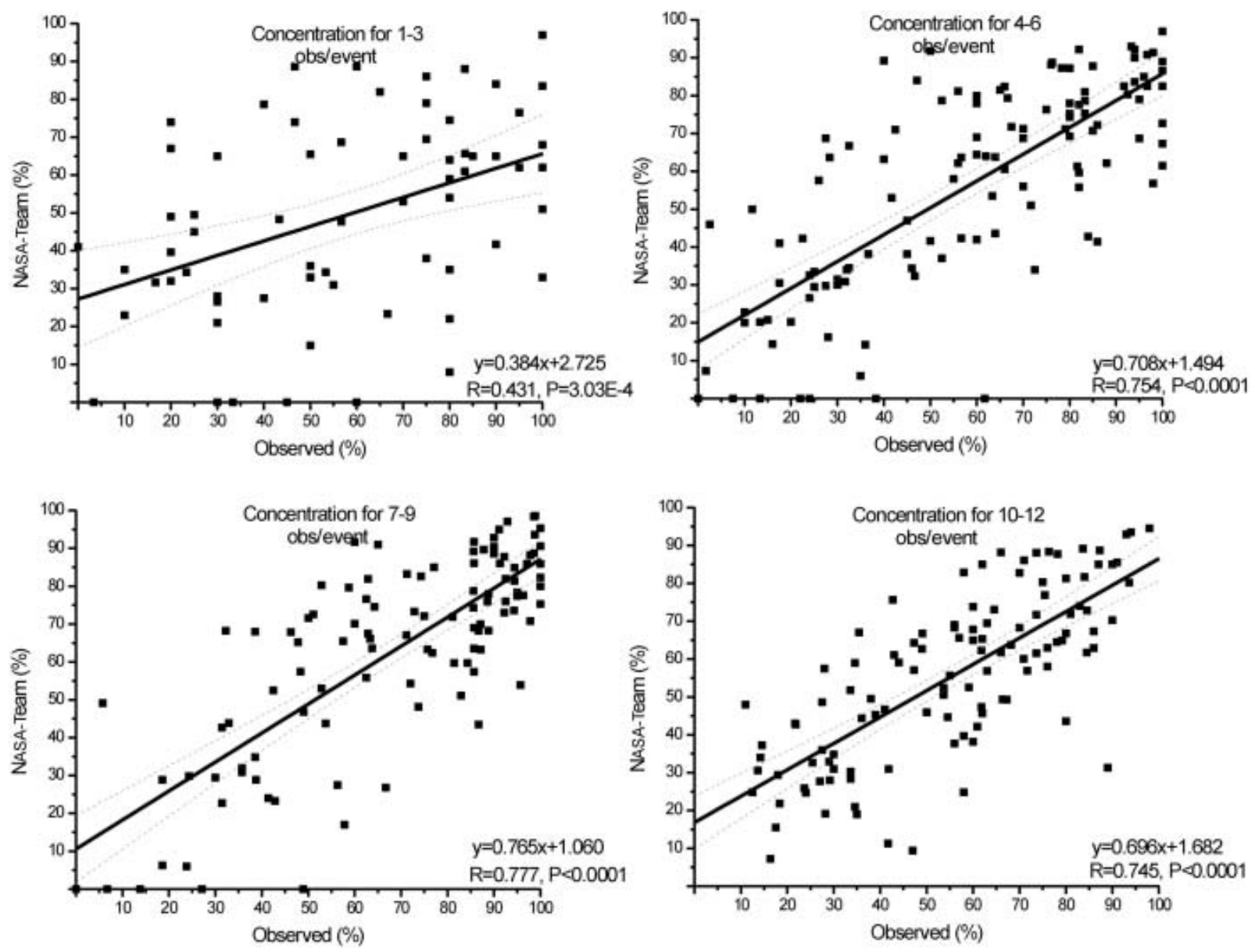

Fig. 3. Observed sea-ice concentration vs ice concentration derived from the NASA-Team algorithm. Each point represents an event, and the plots are separated into how many observations there are per event. The solid line represents a linear fit of the data, and the dashed lines represent $95 \%$ confidence levels. Bootstrap data were similar.

similarity in $R$ values for numbers $>3$ still only explains a maximum of $50 \%$ of the variance (average $R=0.76$ ), with no improvement for the higher number of observations up to 12 . We infer that physical causes are therefore more likely for the unexplained variance between satellite- and videoderived concentrations (beyond 3 observations) than under sampling, as discussed later.

Regardless of the number of observations per event, the most obvious difference in the comparison of the in situ data vs the two algorithms is over areas of known fast ice. As expected, the mean concentration derived from the fast-ice video observations is very high at $95 \%$, while the NASATeam and Bootstrap algorithms had less than half this value, $44 \%$ and $46 \%$, respectively. However, there are several factors that can contribute to inaccurate satellite measurements near the coast. First, there is the land mask being used for the algorithms; some pixels could be designated as ocean when in fact they are actually land and vice versa. There is also the problem of land-to-ocean spillover where, with such a large footprint size from the satellite, contamination can occur due to a blurring of the sharp contrast between land and ocean (Cavalieri and others, 1999). Second, a thick snow cover (>1 m) was reported from the APIS ship data; the satellite is likely to underestimate the actual concentration of the sea ice if influenced, for example, by freshwater ice layers and ice lenses that were also observed in the thick snow covers. Since fast ice was found to extend out from the coastline for several tens of kilometers in some locations (potentially reducing the landice proximity effects), this comparison suggests a stronger effect of the snow cover on microwave brightness temperature in this region, but some role for land-mask (coastline) errors cannot be ruled out. While fast ice makes up only a small percentage of Antarctic ice cover in the summer, it is another problem to consider when dealing with satellite data near the coast.

\subsection{Data by latitude bands}

Sea-ice conditions at the time of the APIS cruise were very complex, and generalized into five zones: northern marginal ice zone (NMIZ), interior pack ice zone (IPIZ), ice-covered shelf zone (ICSZ), the coastal polynya zone (CPZ) and the fast-ice zone. These zones can be estimated by latitude bands where $65-70^{\circ} \mathrm{S}$ represents the $\mathrm{NMIZ}, 71-72^{\circ} \mathrm{S}$ the IPIZ, $73-74^{\circ} \mathrm{S}$ the ICSZ, and $75-77^{\circ} \mathrm{S}$ the CPZ. APIS cruise observations described the two southern zones (CPZ and ICSZ) as dominated by thick and substantially ridged multiyear ice (when ice was present), while the NMIZ and IPIZ ice covers were mostly $<1$ year old, with less ridging and snow cover than floes in the southern zones (Ackley and others, 2003). For continuity, the latitude bands and zone names as described for the APIS cruise were adopted for the AnSlope datasets as well, although we expect to encounter less multi-year ice in the higher latitudes of the western Ross Sea. This resulted in 78, 135, 91 and 36 total events for each zone respectively. To examine the satellite algorithms' 

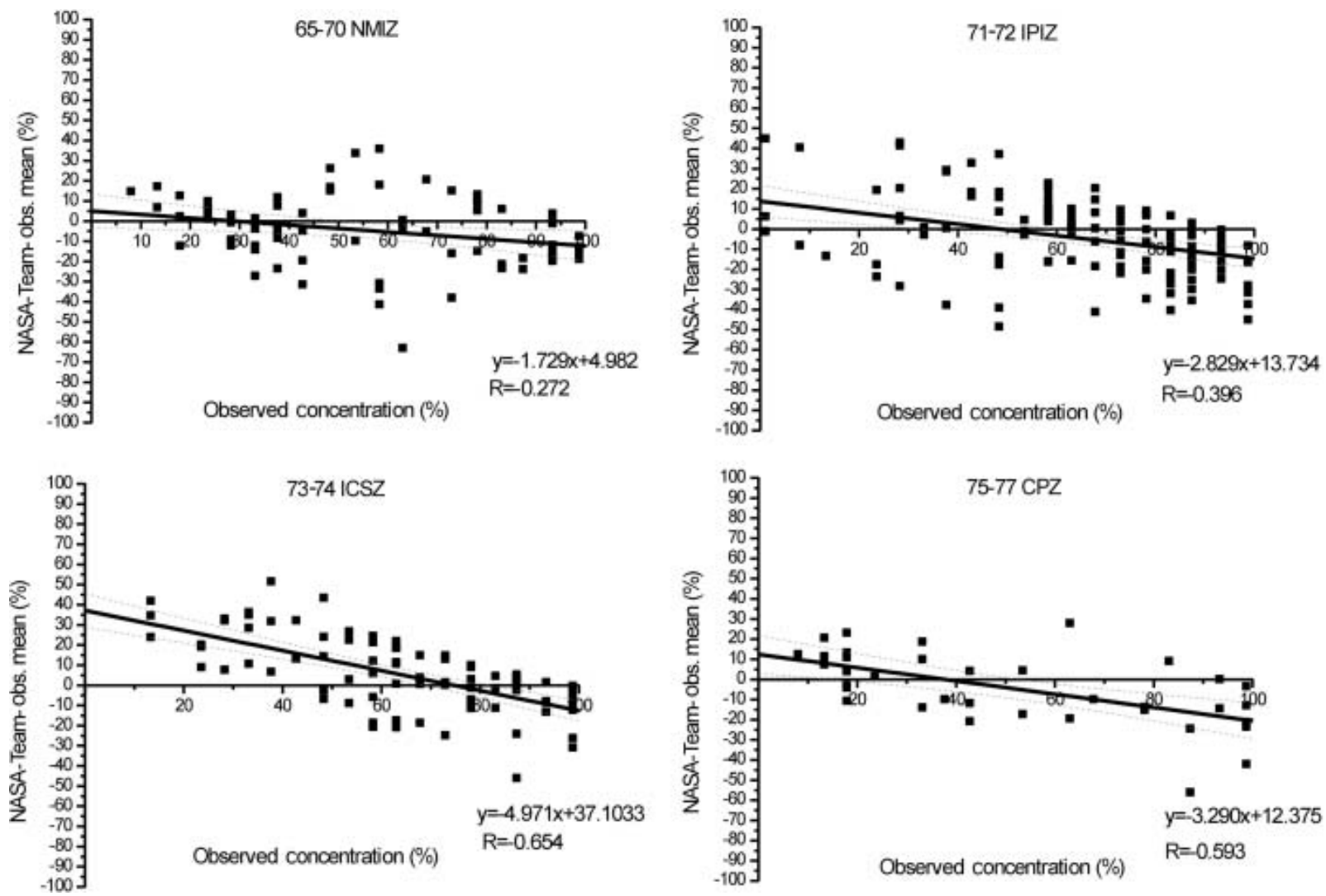

Fig. 4. Trends at varying latitudes of the observed sea-ice concentration vs the ice concentration derived from NASA-Team algorithm minus the mean of the observed data. The solid line represents a linear fit of the data, and the dashed lines represent $95 \%$ confidence levels.

ability to predict, ice-concentration plots of observed vs satellite values minus the observation mean (of a $5 \%$ bin) were made (Fig. 4). Plotting the data in this way allows us to see trends of over- and underestimation by the satellite data, as, even for highly scattered data, a linear fit with zero trend would lie along the $x$ axis of the plot. However, for example, for the plot of $65-70^{\circ}$, the linear fit of the data crosses from positive to negative at a concentration of $30 \%$. Therefore, we can say that in this area the NASA-Team algorithm is under-predicting in areas with an observed ice concentration greater than $30 \%$ and over-predicting for lower concentrations of $<30 \%$. In the $71-72^{\circ}$ (IPIZ) zone, the crossover point occurs at a concentration of about $50 \%$ and for $73-74^{\circ}$ (ICSZ) at a concentration of $75 \%$. For the $75-77^{\circ}$ zone, the cut-off between the two regions is back down to a concentration of approximately $37 \%$. Though there are fewer events to analyze in this region, since the CPZ was documented as having older, thick snow-covered floes, it is possible that the condition of the surface is distorting its microwave signature. For the Bootstrap algorithm, the IPIZ crosses at a concentration of roughly $55 \%$, the ICSZ at the high concentration of $85 \%$, and the CPZ crosses over at $40 \%$. However, for the NMIZ, the linear fit of the data never crosses the axis, and thus under-predicts the ice concentration, if only slightly, $100 \%$ of the time. Figure 5 presents the entire NASA-Team dataset. The linear fit line crosses at just greater than $50 \%$ concentration, and at the ends (concentrations of 0 and $100 \%$ ) the algorithm seems to over- and underestimate by approximately 15\%. The Bootstrap algorithm is slightly different, in that the crossover point is just less than $60 \%$ and it over- and underestimates by about $10 \%$ on average.
Several points are located well outside what would be considered an acceptable amount of error. These outliers, defined in this case to be points with a difference in concentration of $> \pm 20 \%$, can be found in all of the four zones. This produced 89 outlier events for the NASA-Team algorithm and 79 for the Bootstrap algorithm, about a quarter of the total number of events in both cases. This can also be broken down into latitudes and we find that in the NASA algorithm $24 \%$ of the NMIZ, $27 \%$ of the IPIZ, 30\% of the ICSZ and $19 \%$ of the CPZ are outliers. Similarly, the Bootstrap is $19 \%, 26 \%, 25 \%$ and $17 \%$ respectively. Each

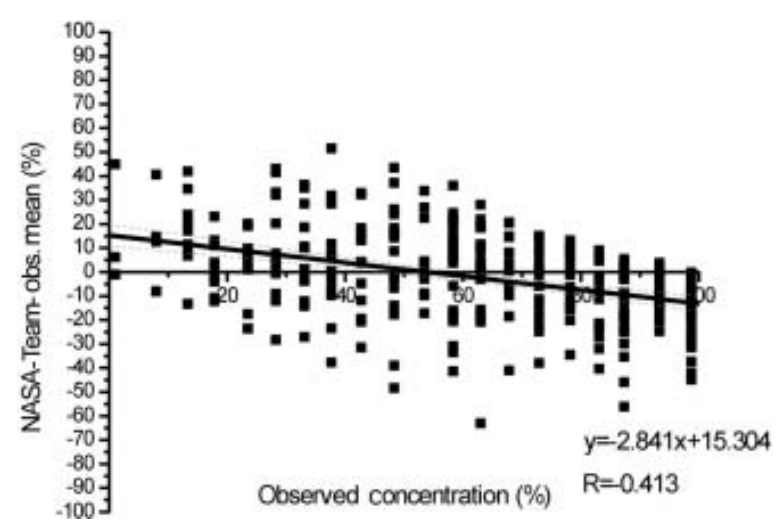

Fig. 5. The observed sea-ice concentration vs the ice concentration derived from NASA-Team algorithm minus the mean of the observed data. This plot represents data from all four of the regions in Figure 4 combined. The solid line represents a linear fit of the data, and the dashed lines represent 95\% confidence levels. 

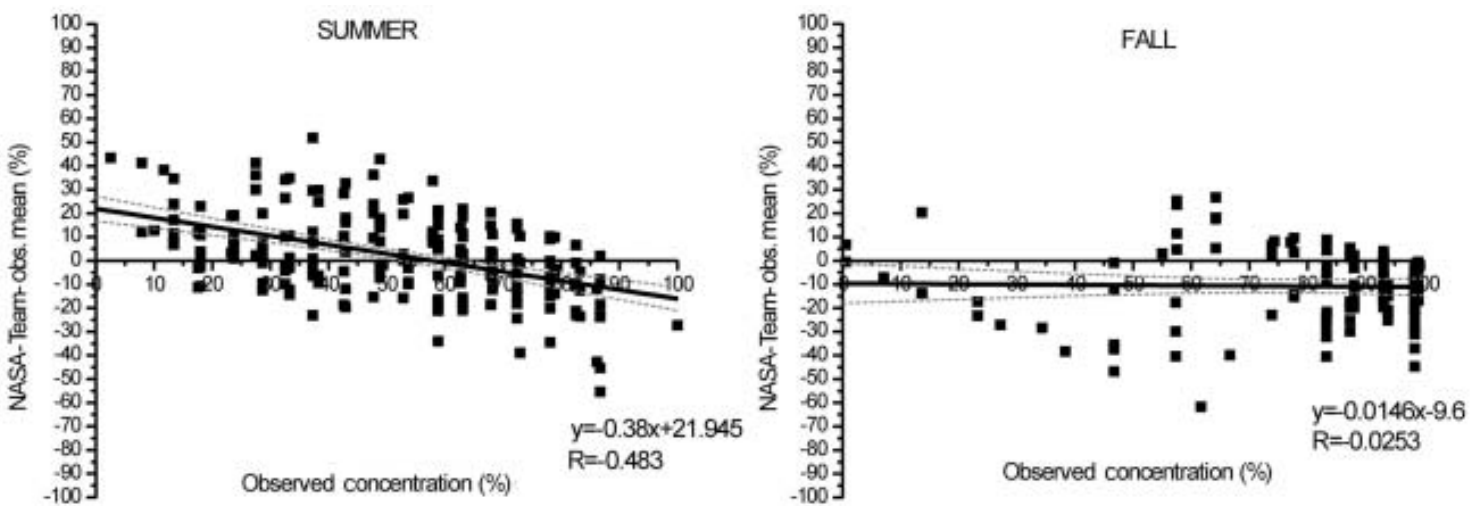

Fig. 6. Trends by season of the observed sea-ice concentration vs the ice concentration derived from NASA-Team algorithm minus the mean of the observed data. The solid line represents a linear fit of the data, and the dashed lines represent $95 \%$ confidence levels.

event's characteristic ice type, floe size, topography and snow cover were also evaluated. $60 \%$ of the NASA-Team and $67 \%$ of the Bootstrap outlier events were thick first-year or multi-year floes. Approximately $60 \%$ of those outliers also had substantial $(>50 \mathrm{~cm})$ snow cover. For both algorithms, when the satellite underestimated the observed values, there were thin secondary and/or tertiary ice types also recorded in the region (NASA-Team 77\% and Bootstrap 79\%). Neither of these datasets exhibited a particular pattern related to the floe size or topography. Although the video data cannot be analyzed for ice surface flooding beneath the snow cover, ship observations and surface data collected concurrently during the APIS cruise showed extensive evidence for seawater flooding at the snow-ice interface for $\sim 80 \%$ of the ice-cover area, as recorded in the ice observations dataset, suggesting that surface flooding may also be an issue for satellite under-prediction at higher concentrations.

\subsection{Data separated into summer and early fall}

Seasonal comparisons of the data were also made. Based on the timing of the cruises involved, we have split the data into two 'seasons.' Summer is defined as all data from December to mid-February, while fall data are from mid-February to April. While calling mid-February 'fall' may seem a little early, brightness temperature data show a distinct change at that time (Willmes and others, 2006). Their data show that higher daily scatter occurs during the summer months (December and January), with a distinct change in measured brightness temperature around mid-February. At that time, they show there is both a reduction in scatter and a drop in the overall brightness temperature. This finding lends support to our statement that mid-February is the beginning of a seasonal change (summer to fall) in the surface characteristics of the sea ice as characterized by brightness temperature. Potentially this could be caused by a reduction in diurnal effects that minimizes daily change or it could simply be from any melt ponds or wet areas on the surface freezing up.

Looking again at the observed value vs the NASA-Team algorithm-observed value, there is a very obvious difference between the summer and fall graphs (Fig. 6). For the summer, the general trend follows the over- and underpredicting that has been shown previously, with a crossover point of about $57 \%$. However, in the fall we see that the trend is to under-predict at any concentration. Only the NASA-Team algorithm is shown here, but the Bootstrap algorithm has similar results. For the fall data, the linear fit for plots of observed data vs satellite-derived values has a higher $R$ value $(\sim 0.85$ for both algorithms, or explainable variance of $72 \%$ ) than the $R=0.7$ (explainable variance $<50 \%$ ) that describes the entire dataset as explained in section 3.1.

\section{CONCLUSIONS}

In situ sea-ice data taken during three cruises aboard the RVIB Nathaniel B. Palmer and from its helicopters (for one of the cruises only) were compared with ice concentration derived from satellite passive microwave data from the same period using the NASA-Team and Bootstrap algorithms. To verify the use of the 'modified' ASPeCt protocol on the helicopter video data, nine whole videos were processed on the computer to find total ice concentration. The difference between the 'modified' ASPeCt point-sampling method for finding average concentration and ice concentration obtained from near-continuous digitally processed images had a small standard deviation $( \pm 3.3 \%)$. While helicopter/video surveillance of ice conditions made by covering a more extensive region than the ship alone is advantageous, the point-sampling method of the ASPeCt protocol for both ship observations and as modified for helicopter image sampling provides a reasonably accurate estimate of the ice concentration over the area surveyed.

Cruise observations illustrated the complex nature of the Ross Sea in the summer months, and comparison with satellite data demonstrates the algorithms' capabilities in calculating sea-ice concentration. Both algorithms tend to generally underestimate concentrations in the NMIZ and also close to shore in the CPZ. The NMIZ is often a zone of warmer ice in summer, and the algorithms' inability to make accurate predictions here may be related to surface flooding and/or the presence of melt-freeze cycles in the snow, which cause the variability in brightness temperatures (Willmes and others, 2006). A serious underestimate was also apparent in the fast-ice cases, with cause somewhat indistinguishable between the presence of thick snow and land-mask (coastline) errors in the microwave imagery. In the IPIZ, the prediction was to over- and then underestimate almost equally as the concentration increased. For the ICSZ, there was a tendency to overestimate the concentration until it reached a value of $70 \%$. These two regions also had the highest incidence of events with a high degree of error. 
Nihashi and others (2005) also found differences (sometimes large) between ASPeCt observations and satellitederived data and concluded that floe size and resolution were the contributing factors. While resolution is an issue (as previously discussed), the extensive helicopter video dataset removes the bias related to floe size and leaves us to believe physical characteristics of the ice and snow (thus emissivity) are responsible for differences in the compared ice concentrations.

One difference between the two algorithms is the percentage of over- and under-prediction at the extremes. As described earlier, estimates are that the ASPeCt protocol has ice concentration errors (from rounding and observer error) up to $10 \%$. This is within the range of the Bootstrap maximum/minimum error, but not the NASA-Team error of up to $15 \%$. The seasonal analysis showed a pattern of overand under-predicting for the summer months when the seaice surface is known to be very complex, and the trend was to consistently under-predict for the fall data. This difference in the fall could be due to the changing (freezing) conditions in the snow and on the ice surface. In any case, this seasonal difference would make studying the annual variation of the concentration difficult. With new instruments, there will be enhancements to both of these algorithms. These changes should be carefully studied over a variety of validation sites for different seasons. However, for historical data and comparing between the old and new instruments, it is important to know how accurate the sea-ice concentrations from the SSM/I really are. The crossover from underpredicting to over-predicting concentrations, and the variability of that crossover point, gives higher error when using microwave imagery in looking, for example, at the interannual variations in summer concentration (December and January) in this region compared to better comparisons that can be made in colder conditions prevailing through the rest of the year.

The satellite data used in this study were provided at the NSIDC website. Since the study was completed, we have learned that there are actually two datasets of SSM/I ice concentration provided on this website. The first, from NSIDC, uses standard tie points, a standard weather filter and that does not remove pixels with coastal contamination. The second, provided by the Goddard Space Flight Center (GSFC) to NSIDC, uses adjusted tie points, advanced weather filtering, and purports to remove data subject to coastal contamination. This study used the first dataset, so it would be an interesting future study to see if the differences found here would be reduced with the GSFC dataset. Such a study may especially resolve the effects of snow cover vs coastal contamination in examination of the fast-ice correlation that we were not able to determine. Correlation of these in situ datasets with both of these publicly available satellite datasets, with the quantifiable errors this correlation provides, may also give the user community a better basis for choosing one or other satellite dataset available at NSIDC.
Regardless of the datasets used in this work, the difficulty encountered during summer retrievals of sea-ice concentration has been highlighted and the authors hope this work will provide some information for the planning needed for future validation studies.

\section{ACKNOWLEDGEMENT}

This research was funded by the US National Science Foundation under grants OPP-9814968 and OPP-9908694.

\section{REFERENCES}

Ackley, S.F. and 14 others. 2003. A top-down, multidisciplinary study of the structure and function of the pack-ice ecosystem in the eastern Ross Sea, Antarctica. Polar Rec., 39(3), 219-230.

Cavalieri, D.J., C.L. Parkinson, P. Gloersen, J.C. Comiso and H.J. Zwally. 1999. Deriving long-term time series of sea ice cover from satellite passive-microwave multisensor data sets. J. Geophys. Res., 104(C7), 15,803-15,814.

Comiso, J.C., D.J. Cavalieri, C.L. Parkinson and P. Gloersen. 1997. Passive microwave algorithms for sea ice concentration: a comparison of two techniques. Remote Sens. Environ., 60(3), 357-384.

Gloersen, P., W.J. Campbell, D.J. Cavalieri, J.C. Comiso, C.L. Parkinson and H.J. Zwally. 1992. Arctic and Antarctic sea ice, 19781987: satellite passive-microwave observations and analysis. Washington, DC, National Aeronautics and Space Administration. (NASA SP-511.)

Markus, T. and D.J. Cavalieri. 1998. Snow depth distribution over sea ice in the Southern Ocean from satellite passive microwave data. In Jeffries, M.O., ed. Antarctic sea ice: physical processes, interactions and variability. Washington, DC, American Geophysical Union, 19-39. (Antarctic Research Series 74.)

Massom, R.A., J.C. Comiso, A.P. Worby, V.I. Lytle and L. Stock. 1999. Regional classes of sea ice cover in the East Antarctic pack observed from satellite and in situ data during a winter time period. Remote Sens. Environ., 68(1), 61-76.

Nihashi, S., K.I. Ohshima, M.O. Jeffries and T. Kawamura. 2005. Sea-ice melting processes inferred from ice-upper ocean relationships in the Ross Sea, Antarctica. J. Geophys. Res., 110(2), 1-12.

Steffen, K. and A. Schweiger. 1991. NASA team algorithm for sea ice concentration retrieval from Defense Meteorological Satellite Program special sensor microwave imager: comparison with Landsat satellite imagery. J. Geophys. Res., 96(C12), 21,971$21,987$.

Willmes, S., J. Bareiss, C. Haas and M. Nicolaus. 2006. The importance of diurnal processes for the seasonal cycle of sea-ice microwave brightness temperatures during early summer in the Weddell Sea, Antarctica. Ann. Glaciol., 44 (see paper in this volume).

Worby, A.P. and S.F. Ackley. 2000. Antarctic research yields circumpolar sea ice thickness data. EOS Trans. AGU, 81(17), 181, 184-185.

Worby, A.P. and J.C. Comiso. 2004. Studies of the Antarctic sea ice edge and ice extent from satellite and ship observations. Remote Sens. Environ., 92(1), 98-111. 